\title{
A Framework for Extracted View Maintenance
}

\author{
Besat Kassaie \\ Cheriton School of Computer Science \\ University of Waterloo \\ Waterloo, Ontario, Canada \\ bkassaie@uwaterloo.ca
}

\author{
Frank Wm. Tompa \\ Cheriton School of Computer Science \\ University of Waterloo \\ Waterloo, Ontario, Canada \\ fwtompa@uwaterloo.ca
}

\begin{abstract}
When information extraction programs (extractors) are applied to documents, they create relations that store facts found in the documents. In this work, we formalize and address the problem of keeping such extracted relations consistent with source documents that are arbitrarily updated. We define three classes of document updates, namely those that are irrelevant, autonomously computable, and pseudo-irrelevant with respect to a given extractor. Finally, we propose algorithms to detect pseudo-irrelevant document updates with respect to extractors that are expressed as document spanners, a model of information extraction inspired by System $T$.
\end{abstract}

\section{CCS CONCEPTS}

- Information systems $\rightarrow$ Information extraction; Database views; $\bullet$ Theory of computation $\rightarrow$ Formal languages and automata theory; $\bullet$ Applied computing $\rightarrow$ Document management and text processing.

\section{ACM Reference Format:}

Besat Kassaie and Frank Wm. Tompa. 2020. A Framework for Extracted View Maintenance. In ACM Symposium on Document Engineering 2020 (DocEng '20), September 29-October 2, 2020, Virtual Event, CA, USA. ACM, New York, NY, USA, 4 pages. https://doi.org/10.1145/3395027.3419592

\section{INTRODUCTION}

Document processing systems include three main components: source documents, programs that read and manipulate those documents, and the resulting artifacts produced by those programs. For example, document converters and formatters produce new documents, markup strippers produce pairs of plain text and standoff markup, search engine indexers produce sets of postings lists, and information extraction systems produce relational tables. Most such systems share a subtle and important assumption that we call "fading attachment." The flow of information between the three main components-source documents, programs, and the resulting artifacts-is maintained during the program development period but evaporates when the program reaches a satisfactory level of

We gratefully acknowledge financial assistance received from the University of Water loo and NSERC, the Natural Sciences and Engineering Research Council of Canada.

Permission to make digital or hard copies of all or part of this work for personal or classroom use is granted without fee provided that copies are not made or distributed for profit or commercial advantage and that copies bear this notice and the full citation on the first page. Copyrights for components of this work owned by others than ACM must be honored. Abstracting with credit is permitted. To copy otherwise, or republish, to post on servers or to redistribute to lists, requires prior specific permission and/or a fee. Request permissions from permissions@acm.org.

DocEng '20, September 29-October 2, 2020, Virtual Event, CA, USA

(c) 2020 Association for Computing Machinery.

ACM ISBN 978-1-4503-8000-3/20/09...\$15.00

https://doi.org/10.1145/3395027.3419592 accuracy and robustness. Once deployed, document processing systems ignore the relationship between the contents of the source documents and the resulting artifacts.

Instead, we can consider the resulting artifacts to be parts of a materialized view of the document corpus. From this perspective, keeping materialized artifacts in sync with the document corpus as documents are updated resembles the problem of maintaining materialized views in relational database systems [7].

As in the relational database context, re-applying a document processor from scratch after each document update can be costly. For instance, in some applications where updates to source documents occur frequently, processing time might be a bottleneck or, in a distributed setting in which the resulting artifacts reside in different physical sites from those housing the source documents, the communication cost for repeatedly transferring newly produced artifacts might be significant. Thus, avoiding re-processing is sometimes highly desirable

The problem of maintaining materialized artifacts has been studied extensively in the relational database setting. Based on the requirements of target applications and the nature of the updates, proposed solutions range from recomputing views from scratch to detecting irrelevant and autonomously computable updates [1] and to updating views differentially $[8,12]$ or only as needed $[4,16]$. Similar approaches have rarely been applied to document processing systems, but Schmidt has proposed an algorithm for differentially updating standoff markup [15] and Chen et al. have developed an approach for differentially updating extracted relations [3]. Other optimization techniques can also be adopted from relational databases [14], including the materialization of partially extracted views (which would also need to be maintained, of course). In fact, we hypothesize that any of the proposed solutions in the relational setting can be adapted to the problem of maintaining artifacts from document processing systems.

Like Chen et al. [3], we wish to adapt the concept of maintaining materialized views from relational database management systems to information extraction systems. How to push back updates to relations that are extracted from documents to updates on the source documents themselves was introduced and formalized last year [10]. In this paper we address the reverse problem for extractors, i.e., determining when we can avoid re-applying extractors after documents are updated (Figure 1).

Given a collection of documents $\mathbb{D}$, a set of extraction programs $\mathbb{E}$, a corresponding set of extracted relations $\mathbb{R}$, and an instance of a document update specification $U$, we study conditions under which we can apply $U$ to members of $\mathbb{D}$ and apply corresponding updates to members of $\mathbb{R}$ without recomputing the revised extracted relations from scratch. That is, we wish to translate updates over 


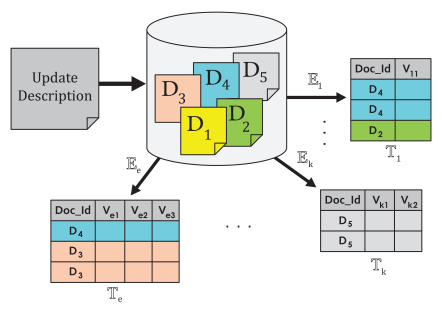

Figure 1: Extraction system that supports updates to all members of a document database.

documents into differential updates over extracted relations. Thus, in this paper:

(1) We introduce the extracted view maintenance problem.

(2) We propose a simple match-and-replace document update model.

(3) We formalize three categories of document updates for which we can preserve consistency without repeating the extraction process.

(4) We propose algorithms to determine whether an update is pseudo-irrelevant with respect to extractors expressed as document spanners, a formalism that models the basis of the SystemT extraction system [14].

\section{EXTRACTORS VIA DOCUMENT SPANNERS}

In order to develop specific algorithms, we assume that extracted views are defined using SystemT, an information extraction platform that benefits from relational database concepts to deal with text data sources $[14]^{1}$. SystemT models each document as a single string and populates relational tables with spans, directly extracted from the input document. With SystemT users encode extractors with a SQL-like language, i.e., AQL, to manipulate tables. AQL offers operators to work directly on text or on the extracted tables (standard relational operators that accept span predicates).

The underlying principles adopted by SystemT have been formalized as document spanners by Fagin, et al. [6]. Most of the material in this section has been introduced in that work, which contains additional details.

Let $\Sigma$ be a finite alphabet and $D$ be a (finite) document over $\Sigma$, i.e., $D \in \Sigma^{*}$. A span of $D$, denoted $[i, j\rangle(1 \leq i \leq j \leq|D|+1)$, specifies the start and end offsets of a substring in $D$, which is in turn denoted $D_{[i, j\rangle}$, and extends from offset $i$ through offset $j-1$. If $i=j$, this denotes an empty span at offset $i$. Spans $s_{1}=\left[i_{1}, j_{1}\right\rangle$ and $s_{2}=\left[i_{2}, j_{2}\right\rangle$ are identical if and only if $i_{1}=i_{2}$ and $j_{1}=j_{2}$; they overlap if $i_{1} \leq i_{2}<j_{1}$ or $i_{2} \leq i_{1}<j_{2}$.

Regular expressions extended using variables chosen from a set $V$ are called regular expressions with capture variables, defined by $\gamma$ in the grammar $G_{S}(\Sigma, V)$ as follows:

$$
\gamma:=\emptyset|\epsilon| \sigma|(\gamma \vee \gamma)|(\gamma \bullet \gamma)\left|(\gamma)^{*}\right| x\{\gamma\}
$$

where $\sigma \in \Sigma$ and $x \in V$. The use of a sub-expression of the form $x\{g\}$ is to denote that whenever the regular expression matches a

\footnotetext{
${ }^{1}$ How to maintain extracted views efficiently should also be investigated using other extraction languages, such as JAPE [5].
}

string, sub-strings matched by $g$ are to be marked by the capture variable $x$. If $E$ is a regular expression with capture variables, then we denote the set of capture variables in $E$ as $S \operatorname{Vars}(E)$. We use $G_{S}$ in place of $G_{S}(\Sigma, V)$ whenever $\Sigma$ and $V$ are immaterial or understood from the context. We also allow regular expressions with capture variables to be written without parentheses that can be inferred based on priority of operations [9].

Applying an information extractor to a document $D$ produces a span relation, i.e., a relation that contains spans of $D$. To this end, if $E$ is a regular expression with capture variables, it specifies a document spanner, denoted $\llbracket E \rrbracket$, which is a function mapping strings over $\Sigma^{*}$ to span relations. In particular, for a given document $D$, the spanner specified by $E$ produces a span relation $\llbracket E \rrbracket(D)$ in which there is one column for each variable from $V$ appearing in $E$, each row corresponds to a matching of $E$ against $D$ when the variables are ignored, and the value in a row for the column corresponding to $x \in V$ is the span marked by $x$. To ensure that the extracted relation is in first-normal form with no null values, we restrict our attention to a specific class of document spanners, namely functional document spanners, that assign exactly one span to each variable for all produced rows, regardless of the input document $D$.

As an example, let $\Sigma$ be the set of alphanumeric characters plus punctuation and the space character (the last represented by $\sqcup$ ), and let $d$ denote a digit. Applying

$$
\begin{aligned}
\gamma_{\text {phone }}=\Sigma^{*} \bullet \operatorname{tn}\{ & (0 \bullet 1 \vee 1 \vee+\bullet 1) \bullet-\bullet a c\{d \bullet d \bullet d\} \bullet \\
& -\bullet d \bullet d \bullet d \bullet-\bullet s c\{d \bullet d \bullet d \bullet d\}\} \bullet \Sigma^{*}
\end{aligned}
$$

to the document in Figure 2 results in the span relation in Figure 3.

In this paper we hypothesize systems that include a document database $\mathbb{D}$ and a set of extractors $\left\{\mathbb{E}_{1}, \cdots, \mathbb{E}_{e}\right\}$ that run independently over $\mathbb{D}$. The union of span relations produced by an extractor $\mathbb{E}_{k}$ against the document database is stored in a relation $\mathbb{T}_{k}$ that includes an additional column to associate each document identifier with the spans for the corresponding span relation, as in Figure 1.

\section{SPANNER-BASED UPDATE MODEL}

We assume that target points of change in a document are specified using patterns over the input string, expressed as a functional document spanner with precisely one variable. Specifically, an update formula is an extraction formula for specifying an update, defined by $\gamma$ in the following grammar $G_{U}(\Sigma, x)$ :

$$
\begin{aligned}
& \gamma:=(\gamma \vee \gamma)\left|\left(\gamma^{\prime} \bullet \gamma\right)\right|\left(\gamma \bullet \gamma^{\prime}\right) \mid x\left\{\gamma^{\prime}\right\} \\
& \gamma^{\prime}:=\emptyset|\epsilon| \sigma\left|\left(\gamma^{\prime} \vee \gamma^{\prime}\right)\right|\left(\gamma^{\prime} \bullet \gamma^{\prime}\right) \mid\left(\gamma^{\prime}\right)^{*}
\end{aligned}
$$

(i.e., where $\gamma^{\prime}$ is a standard, variable-free regular expression).

The functional document spanner that is represented by an update formula $g$ maps every document $D$ to a unary span relation, which we call the update relation and denote as $\llbracket g \rrbracket(D)$. When the spanner is used for updating a document $D$, we require that all spans in $\llbracket g \rrbracket(D)$ be mutually disjoint. In this case, sub-strings of $D$ associated with the spans in the update relation are simultaneously replaced by a new value denoted by a constant $A$. Because the update relation contains non-overlapping spans, such replacements will be mutually non-interfering.

Definition 3.1. An instance of an update specification with given update formula $g$ and $A \in \Sigma^{*}$ is called an update expression and 


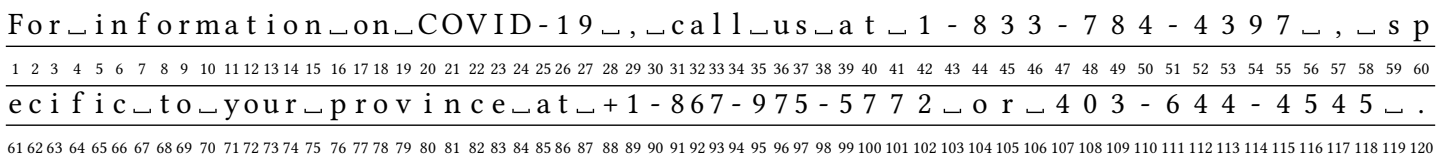

Figure 2: A sample input document $D$ for our running example.

\begin{tabular}{|c|c|c|}
\hline & $\mathrm{ac}$ & $\mathrm{sc}$ \\
\hline$[42,56\rangle$ & {$[44,47\rangle$} & {$[52,56\rangle$} \\
\hline 88,103 & 19 & \\
\hline
\end{tabular}

Figure 3: $\llbracket \gamma_{\text {phone }} \rrbracket(D)$, where $D$ is depicted in Figure 2.

represented by $\operatorname{Repl}(g, A)$. Given a document $D$, if $\llbracket g \rrbracket(D)$ contains no overlapping spans, then applying $\operatorname{Repl}(g, A)$ to $D$ produces a new document $\operatorname{Repl}(g, A)(D)$ that is identical to $D$ but with every substring in $D$ marked by $x$ in $\llbracket g \rrbracket$ replaced by the string $A$.

Note that if $A$ is the empty string, then the update results in the deletion of the substrings identified by the spanner; otherwise, wherever the spanner produces an empty span, the replacement, in effect, inserts the string $A$. For example, given $D$ as in Figure 2, applying $\operatorname{Repl}\left(\Sigma^{*} \bullet \mathrm{u} \bullet \mathrm{s} \bullet \bullet \bullet x\{\epsilon\} \bullet \mathrm{a} \bullet \mathrm{t} \bullet \Sigma^{*}\right.$, free $\left.)\right)$ to $D$ inserts 'free,', at $[39,39\rangle$.

\section{IRRELEVANT AND AUTONOMOUSLY COMPUTABLE UPDATES}

Given an update expression and an extraction spanner, we wish to determine, for all potential input documents, whether the extracted materialized view can be kept consistent with the updated source documents without running the extractor after updating the documents in the database. This problem is similar to filtering out irrelevant updates or applying updates autonomously to relational materialized views [2].

Definition 4.1. An update expression $\operatorname{Repl}(g, A)$ is irrelevant with respect to an extractor $\llbracket E \rrbracket$ if for every input document, applying $\llbracket E \rrbracket$ to $\operatorname{Repl}(g, A)(D)$ produces a span relation that is identical to applying $\llbracket E \rrbracket$ to $D$. That is, if $D^{\prime}=\operatorname{Repl}(g, A)(D)$, then $\llbracket E \rrbracket\left(D^{\prime}\right)=$ $\llbracket E \rrbracket(D)$.

If an update expression is relevant with respect to an extractor, it may be that the modification to the extracted relation can be computed without re-running the extractor.

Definition 4.2. An update expression $\operatorname{Repl}(g, A)$ is autonomously computable with respect to an extractor $\llbracket E \rrbracket$ if for every input document, applying $\llbracket E \rrbracket$ to $\operatorname{Repl}(g, A)(D)$ can be computed from the update expression, the update relation, the extraction formula that defines the extraction spanner, and the extracted relation. ${ }^{2}$

Unlike traditional relational views, span relations contain pairs of offsets from input documents, not document content. Thus a span relation might be affected by an update even if the replaced text is not within an extracted span. In particular, replacing a string of one length by a string of another length somewhere in the document

\footnotetext{
${ }^{2}$ Autonomous computability for updates is analogous to determinacy [13] for queries
}

will cause a span somewhere else in the document to shift, even if the content of that span is unaffected.

More specifically, given a document $D$ and the corresponding updated document $D^{\prime}$, if $\llbracket g \rrbracket(D)$ contains no overlapping spans and span $S$ in $D$ is disjoint from all spans in $\llbracket g \rrbracket(D)$, let $\operatorname{shift}(g, A)(S)$ represent the corresponding span in $D^{\prime}$, i.e., the new location of the content of $S$ in $D^{\prime}$. shift $(g, A)(S)$ is shifted from $S$ by an amount that is dependent on the length of $A$ and the lengths of all spans in the update relation that precede $S$ in $D$, as captured by Algorithm 1 .

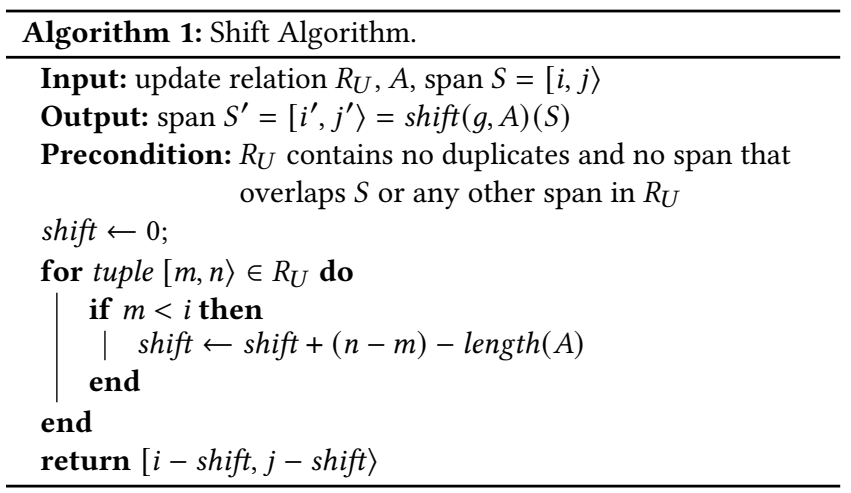

Definition 4.3. Update expression $\operatorname{Repl}(g, A)$ is pseudo-irrelevant with respect to an extraction spanner $\llbracket E \rrbracket$ if for every input document, applying $\llbracket E \rrbracket$ to $\operatorname{Repl}(g, A)(D)$ produces a span relation that is identical to applying $\llbracket E \rrbracket$ to $D$ except to replace each span $S$ by $\operatorname{shift}(g, A)(S)$. That is, if $D^{\prime}=\operatorname{Repl}(g, A)(D)$, then $\llbracket E \rrbracket\left(D^{\prime}\right)=$ $\left\{S^{\prime} \mid \exists S \in \llbracket E \rrbracket(D)\right.$ such that $\left.S^{\prime}=\operatorname{shift}(g, A)(S)\right\}$.

Thus, a pseudo-irrelevant update is a special case of an autonomously computable update.

Note. By definition, if an update expression is irrelevant with respect to an extraction spanner, then it is also pseudo-irrelevant with respect to that spanner.

\section{TESTING FOR PSEUDO-IRRELEVANCE}

We wish to identify whether an update is irrelevant or pseudoirrelevant with respect to a given extractor, independently of input documents. The essence of our approach is to inspect various kinds of overlap between an update expression and an extractor.

If an update changes the content length of an extracted span, then it will be relevant; the extractor should be re-executed. ${ }^{3}$ However, even without changing an extracted value, an update could change

\footnotetext{
${ }^{3}$ There are some conditions under which the extracted relation after update might be autonomously computable. We leave the determination and detection of such conditions for future work.
} 


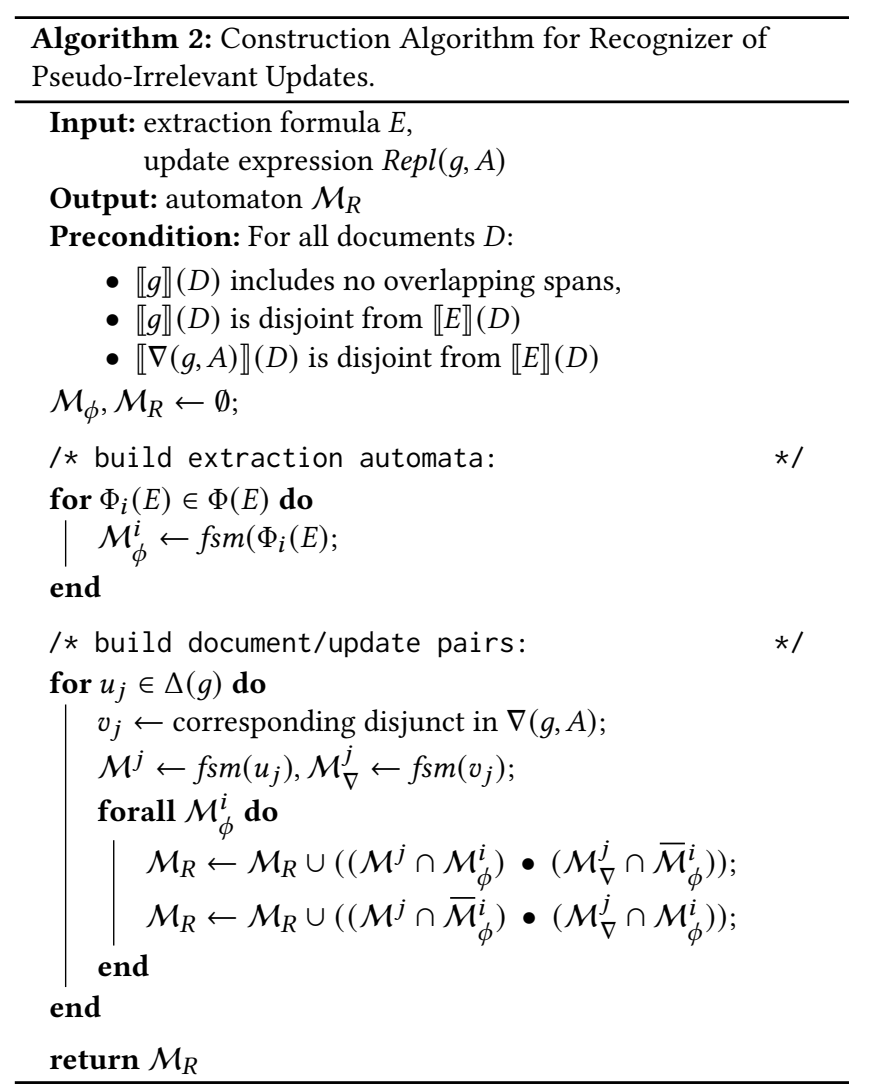

the context for determining that a span should be extracted. First, updated spans, with the new value A, could form new matches for the extraction spanner, which would create new rows in the extracted view if we re-run the extractor. Second, some extracted spans might no longer match after the update, and therefore the associated rows would disappear when the extractor is re-run after the update. ${ }^{4}$

Given any extraction formula $E$, it can be rewritten as $\Delta(E)=$ $\bigvee_{i=1}^{k} E_{i}$, where within each $E_{i}$, all operands for disjunction and Kleene closure are standard, variable-free regular expressions. ${ }^{5}$ The disjuncts can then be partitioned by their variable-profiles, that is, by their specific sequence and nesting pattern of capture variables. Let $\Phi_{i}(E)$ be the disjunction of all disjuncts in the $i^{t h}$ such partition, and let $\Phi(E)=\left\{\Phi_{i}(E)\right\}$.

Definition 5.1. Given $\operatorname{Repl}(\gamma, A)$, the proxy language $\nabla(g, A)$ is defined using the following disjunctive form: $\nabla(g, A)=\bigvee_{i=1}^{k} V_{i}$ where $V_{i}$ is derived from disjunct $U_{i}$ in $\Delta(g)$ by replacing the marked subexpression in that disjunct by $x\{A\}$.

Algorithm 2 creates a machine, i.e., $\mathcal{M}_{R}$, to recognize pseudoirrelevant updates using standard operators including concatenation $(\bullet)$, union $(\cup)$, intersection $(\cap)$, and complement $(\bar{M})$ [9]. (We assume that the built-in function $f s m()$ eliminates all capture variables from an input regular formula and converts the result to its equivalent finite state machine.) Each precondition can be tested by constructing an automaton that matches all witness documents denying the property; thus if the constructed automata are empty, the precondition holds for all documents.
THEOREM 5.2. Let the min function represents standard state minimization. $\min \left(\mathcal{M}_{R}\right) \neq \emptyset$ iff $\exists a$ witness $D$ showing that the unrestricted spanner defined by $\operatorname{Repl}(g, A)$ is not pseudo-irrelevant with respect to the disjoint extractor $\llbracket E \rrbracket$.

\section{FUTURE WORK}

We have established some sufficient conditions for updates to be pseudo-irrelevant, but we have not yet investigated whether there are necessary conditions as well. Furthermore, we have not yet investigated other autonomously computable conditions, such as those that might result in span modifications or insertions of extracted tuples. We have also not yet explored the practicality of constructing our verification automata nor investigated update properties of extractors that are defined by mechanisms more expressive than spanners.

Our model for document updates is quite limited. In practice, correlated updates require that update spanners include several variables, and substituted values often depend on the relative position of the update, some associated string values, or the contexts of matched spans. Loosening these restrictions creates new research challenges for verifying pseudo-irrelevance and other update properties.

${ }^{4}$ These effects are not mutually exclusive.
${ }^{5}$ The extended version [11] has further details and proofs for all material in this section.

\section{REFERENCES}

[1] José A. Blakeley, Neil Coburn, and Per-Åke Larson. 1989. Updating Derived Relations: Detecting Irrelevant and Autonomously Computable Updates. ACM Trans. Database Sys. 14, 3 (1989), 369-400.

[2] José A. Blakeley, Per-Åke Larson, and Frank Wm. Tompa. 1999. Efficiently Updating Materialized Views. In Materialized Views: Techniques, Implementations, and Applications. MIT Press, 163-175.

[3] Fei Chen, AnHai Doan, Jun Yang, and Raghu Ramakrishnan. 2008. Efficient Information Extraction over Evolving Text Data. In ICDE 2008. 943-952.

[4] Latha S. Colby, Timothy Griffin, Leonid Libkin, Inderpal Singh Mumick, and Howard Trickey. 1996. Algorithms for Deferred View Maintenance. In SIGMOD 1996. 469-480.

[5] Hamish Cunningham, Diana Maynard, and Valentin Tablan. 2000. JAPE: a Java annotation patterns engine (second ed.). Technical Report CS-00-10. Dept. Comp. Sci., Univ. Sheffield.

[6] Ronald Fagin, Benny Kimelfeld, Frederick Reiss, and Stijn Vansummeren. 2015. Document Spanners: A Formal Approach to Information Extraction. F. ACM 62, 2 (2015), 12:1-12:51.

[7] Ashish Gupta and Iderpal Singh Mumick (Eds.). 1999. Materialized Views: Techniques, Implementations, and Applications. MIT Press.

[8] Ashish Gupta, Inderpal Singh Mumick, and V. S. Subrahmanian. 1993. Maintaining Views Incrementally. In SIGMOD 1993. 157-166.

[9] John E. Hopcroft, Rajeev Motwani, and Jeffrey D. Ullman. 2007. Introduction to automata theory, languages, and computation (3rd ed.). Addison-Wesley.

[10] Besat Kassaie and Frank Wm. Tompa. 2019. Predictable and Consistent Information Extraction. In DocEng 2019. 14:1-14:10.

[11] Besat Kassaie and Frank Wm. Tompa. 2020. Detecting Opportunities for Differential Maintenance of Extracted Views. (2020). arXiv:2007.01973

[12] Akira Kawaguchi, Daniel F. Lieuwen, Inderpal Singh Mumick, and Kenneth A. Ross. 1997. Implementing Incremental View Maintenance in Nested Data Models. In DBPL-6. 202-221.

[13] Alan Nash, Luc Segoufin, and Victor Vianu. 2010. Views and queries: Determinacy and rewriting. ACM Trans. Database Syst. 35, 3 (2010), 21:1-21:41.

[14] Frederick Reiss, Sriram Raghavan, Rajasekar Krishnamurthy, Huaiyu Zhu, and Shivakumar Vaithyanathan. 2008. An Algebraic Approach to Rule-Based Information Extraction. In ICDE 2008. 933-942.

[15] Desmond Allan Schmidt. 2016. Using standoff properties for marking-up historical documents in the humanities. it - Inf. Technol. 58, 2 (2016), 63-69.

[16] Jingren Zhou, Per-Åke Larson, Jonathan Goldstein, and Luping Ding. 2007. Dynamic Materialized Views. In ICDE 2007. 526-535. 